\title{
REVISIÓN DE INVESTIGACIONES SOBRE ESCRITURA ACADÉMICA PARA LA CONSTRUCCIÓN DE UN CENTRO DE ESCRITURA DIGITAL EN EDUCACIÓN SUPERIOR
}

\author{
A Review of Research on Academic Writing for the Creation of an Online \\ Writing Center in Higher Education
}

REVUE DE RECHERCHE SUR L'ÉCRITURE ACADÉMIQUE POUR LA CONSTRUCTION D'UN CENTRE D'ÉCRITURE NUMÉRIQUE DANS L'ENSEIGNEMENT SUPÉRIEUR

\section{REVISÃo de PESQUiSA SOBRE A ESCRITA ACADÊMICA PARA A CONSTRUÇÃo DE UM CENTRO DE ESCRITA DIGITAL NO ENSINO SUPERIOR}

\author{
Dora Inés Chaverra Fernández \\ Profesora titular, Facultad de Edu- \\ cación, Universidad de Antioquia, \\ Medellín, Colombia. \\ dora.chaverra@udea.edu.co \\ https://orcid. \\ org/0000-0002-1443-5326

\section{Gerzon Yair Calle-Álvarez} \\ Profesor de cátedra, Facultad de \\ Educación, Universidad de Antioquia, \\ Medellín, Colombia. \\ gerzon.calle@udea.edu.co \\ https://orcid. \\ org/0000-0002-4083-6051
}

\section{Rubén Darío Hurtado Vergara Profesor titular, Facultad de Edu- cación, Universidad de Antioquia, Medellín, Colombia. ruben.hurtado@udea.edu.co https://orcid. org/0000-0001-9496-2865}

\begin{abstract}
RESUMEN
Las investigaciones de naturaleza disciplinar o transdisciplinar centradas en el fortalecimiento de competencias para la escritura académico-científica en la educación superior presentan múltiples divergencias de orden teórico y metodológico, máxime cuando se incluyen variables relacionadas con la cultura académica y el uso de tecnologías digitales. Este artículo expone una revisión de estudios asociados a la escritura académica en la educación superior, que podría contribuir al diseño de un centro de escritura digital. La base documental está constituida por 58 textos, analizados con el lenguaje de programación Python $3.7^{\circ}$, utilizando principalmente la librería de lenguajes naturales (Natural Language Toolkit) y técnicas de análisis del discurso. Los resultados de dicha revisión evidencian la consolidación de dos líneas de investigación: a) los componentes pedagógico-didácticos para la promoción de la escritura académica, y b) el fortalecimiento del aprendizaje de la lengua materna. Estos resultados sugieren que no hay convergencia entre postulados requeridos en el diseño, la fundamentación e implementación de estrategias experimentales para la construcción de un centro digital de escritura, como son: a) los epistemológicos, relacionados con las formas de construcción del conocimiento; b) los pedagógicos, asociados al propósito formativo de la escritura en la ciencia y la cultura, y c) los metodológicos, vinculados a la integración de la escritura con las tecnologías digitales.

Palabras clave: educación superior; escritura académica; centros de escritura digital; tecnologías digitales; tutorías.
\end{abstract}

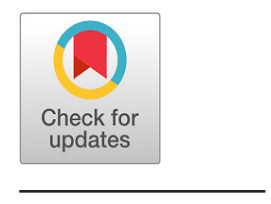

Recibido: 2021-03-13 / Aceptado: 2021-05-27 / Publicado: 2022-02-11

https://doi.org/10.17533/udea.ikala.v27n1a11

Editora: Doris Correa, Universidad de Antioquia, Medellín, Colombia.

Derechos patrimoniales, Universidad de Antioquia, 2022. Este es un artículo en acceso abierto, distribuido según los términos de la licencia Creative Commons BY-NC-SA 4.0 Internacional. 
Wilson Antonio Bolívar Buriticá

Profesor asistente, Facultad de Educación, Universidad de Antioquia,

Medellín, Colombia.

wilson.bolivar@udea.edu.co

https://orcid.

org/0000-0003-2347-1916
Este artículo se deriva de un estudio de caso realizado en pregrados de la Universidad de Antioquia (2019-2020), titulado "Diseño e implementación piloto de un Centro de Escritura Digital en educación superior". El estudio fue financiado con recursos del Sistema General de Regalías y fondos de Ciencia, Tecnología e Innovación (CTel) de la Gobernación de Antioquia, administrados a través del Ministerio de Ciencia, Tecnología e Innovación (Minciencias), Colombia (Contrato 80740-004-2018).

\section{Abstract}

Disciplinary or transdisciplinary research focused on enhancing competencies for academic-scientific writing in higher education present multiple theoretical and methodological divergences, especially when variables related to academic culture and the use of digital technologies are included. This article presents a review of studies associated with academic writing in higher education, which could contribute to the design of a digital writing center. The data are made up of 58 texts, analyzed with the Python $3.7^{\circ}$ programming language, using the Natural Language Toolkit library and discourse analysis techniques. The results of this review show the consolidation of two lines of research: (a) the pedagogical-didactic components for the promotion of academic writing, and (b) strategies to strengthen the learning of the main language. These results suggest that there is no convergence among postulates required in the design, foundation and implementation of experimental strategies for a digital writing center, such as: (a) epistemological postulates, related to the forms of construction of knowledge; (b) pedagogical postulates, associated with the educational purpose of writing in science and culture; and (c) methodological postulates, connected to the integration of writing with digital technologies.

Keywords: higher education; academic writing; digital writing centers; digital technologies; tutoring.

\section{RÉSUMÉ}

La recherche de nature disciplinaire ou transdisciplinaire axée sur le renforcement des compétences pour l'écriture académique et scientifique dans l'enseignement supérieur présente de multiples divergences de nature théorique et méthodologique, en particulier lorsque des variables liées à la culture académique et à l'utilisation des technologies numériques sont incluses. Cet article présente une revue documentaire des études associées à l'écriture académique dans l'enseignement supérieur, qui pourrait contribuer à la conception d'un centre d'écriture numérique. La base documentaire se compose de 58 textes, analysés à l'aide du langage de programmation Python $3.7^{\circ}$, principalement à l'aide de la bibliothèque Natural Language Toolkit et des techniques d'analyse du discours. Les résultats de cette revue montrent la consolidation de deux axes de recherche : a) des études sur les composantes pédagogiques et didactiques pour la promotion de l'écriture académique, et b) le renforcement de l'apprentissage de la langue maternelle. Ces résultats suggèrent qu'il n'y a pas de convergence entre les postulats épistémologiques liés aux formes de construction de la connaissance ; pédagogique, associée à l'objectif formateur de l'écriture en science et culture, et méthodologique, de l'intégration avec les technologies numériques. Tous sont nécessaires à la conception, à la fondation et à la mise en œuvre de stratégies expérimentales telles qu'un centre d'écriture numérique.

Mots-clés : enseignement supérieur ; écriture académique ; centres d'écriture numérique; technologies numériques; tutoriels.

\section{Resumo}

Investigações de natureza disciplinar ou transdisciplinar focalizadas no fortalecimento de competências para a elaboração de material escrito acadêmico-científico no ensino superior apresentam múltiplas divergências teóricas e metodológicas, especialmente quando se incluem variáveis relacionadas à cultura acadêmica e ao 
uso de tecnologias digitais. Este artigo apresenta uma revisão de estudos associados à elaboração de material escrito acadêmico no ensino superior, que podem contribuir para a concepção de um centro de escrita digital. A base documental é composta por 58 textos, analisados na linguagem de programação Python $3.7^{\circ}$, principalmente por meio da biblioteca Natural Language Toolkit e de técnicas de análise de discurso. Os resultados desta revisão mostram a consolidação de duas linhas de pesquisa: 1) os estudos sobre os componentes pedagógico-didáticos para a promoção da elaboração de material escrito acadêmico e 2) o fortalecimento da aprendizagem da língua materna. Esses resultados sugerem que não há convergência entre postulados epistemológicos relacionados às formas de construção do conhecimento; pedagógicos, associada à finalidade educacional da elaboração de material escrito em ciência e cultura, e metodológica, a partir da integração com as tecnologias digitais. Todos eles necessários na concepção, fundação e implementação de estratégias experimentais como um Centro de Escrita Digital.

Palavras chave: ensino superior; elaboração de material escrito; centros de escrita digital; tecnologias digitais; tutorias. 


\section{Introducción}

Un interés de la educación superior es formar a los estudiantes en la lectura y la escritura académica, lo que favorece el aprendizaje, el desarrollo profesional y personal (Al Murshidi y Al Abd, 2014), y la participación en comunidades científicas. El dominar las competencias de lectura y escritura académica permite que el estudiante interprete, analice, argumente, produzca y exprese sus ideas desde los saberes disciplinares, lo que favorece la discusión, la divulgación y el intercambio de información y conocimientos (Ballén Vargas y Herrera González, 2017). Dichas competencias repercuten en un mejoramiento de la calidad de la educación, en la medida en que todas las áreas del conocimiento están mediadas por ellas.

Las formas de lectura y escritura de la universidad difieren de los modelos de la educación secundaria (Carlino, 2017). Esta diferencia es comprensible, porque la producción y la comprensión de textos en la educación básica y media corresponden a prácticas sociales y lógicas acordes con estos niveles educativos, donde el estudiante reconoce, explora y aplica las tipologías textuales que circulan en su espacio académico y social, mientras que en la universidad hay un lenguaje académico y científico propios de las disciplinas, que les permiten a los estudiantes comprender, crear y divulgar los saberes científicos.

El interés de formar en escritura académica ha llevado a la construcción de teorías sobre la enseñabilidad y el aprendizaje de las competencias comunicativas en la educación superior, como la escritura a través del currículo, la escritura en las disciplinas, la alfabetización académica, la posalfabetización y la literacidad crítica. Y desde sus comprensiones y realidades institucionales, se han diseñado estrategias institucionales, como los Academic Skills Centres (Solimine y Marzal García-Quismondo, 2020), los centros de escritura (Calle Arango, 2020; Crowley-Watson, 2019; Peña Portero y Santamaría Martínez, 2020) y programas de lectura y escritura (Molina-Natera y
López-Gil, 2020). Sin embargo, estas estrategias requieren de la enseñanza de la escritura académica desde las disciplinas, por parte de los profesores universitarios, donde el estudiante sea el centro del proceso formativo y se conciba la escritura académica como una competencia susceptible de mejora (Zárate Fabián, 2017). Además, las intervenciones del profesor universitario en el proceso conceptual y lingüístico de los estudiantes debería inducir una reflexión activa en la escritura (Álvarez y Difabio de Anglat, 2018), que es precisamente lo que les permite, por un lado, tomar conciencia tanto de lo que significa escribir como de los diferentes componentes lingüísticos a tener en cuenta en el proceso de la producción textual; y por otro, posibilitar la generación de nuevo conocimiento, como consecuencia de la reflexión sobre lo que se desea comunicar, en diálogo con el campo de conocimiento en el cual se participa.

En la discusión sobre la escritura en la educación superior se identifican diferentes conceptos a ella asociados, como son los de escritura académica, escritura cientifica y escritura disciplinar. Sin embargo, sus alcances y acepciones se encuentran vinculados a propósitos comunicativos, cognitivos, divulgativos o formativos. En general, estos conceptos reflejan una preocupación por el aprendizaje y la enseñanza de la lectura y la escritura en los estudiantes de pregrado y posgrado, para el acceso a los conocimientos académicos y científicos desde un enfoque de proceso, superando el enfoque remedial, desde el cual subsanar los problemas lingüísticos y discursivos que se presentan para producir un texto (Álvarez y Difabio de Anglat, 2018; Hernández Rojas y Rodríguez Varela, 2018; Zárate Fabián, 2017).

Desde este enfoque, se explicita la importancia de los procesos de alfabetización académica durante toda la formación profesional (Carlino, 2013; Navarro et al., 2019). Con ella se alude a formas propias de enseñanza, aprendizaje, apropiación, comunicación y divulgación del conocimiento de una comunidad académica especializada, de la cual las competencias comunicativas, entre ellas, 
leer y escribir, hacen parte. De acuerdo con Carlino (2013, 2017), tanto la lectura como la escritura convierten el conocimiento en objetos para pensar e "incluye a los estudiantes universitarios en situaciones discursivas típicas de comunidades especializadas según propósitos, significados y valores compartidos" (Carlino, 2013, pp. 361-362).

La escritura académica no es el resultado de ideas neutras o abstractas, sino un proceso de construcción social, que atiende a situaciones contextuales, comunicativas y divulgativas (Ortiz Casallas, 2015), proceso que resulta igualmente exigente cuando se relacionan las competencias comunicativas con las demandas de la denominada "sociedad del conocimiento”, en la cual las tecnologías digitales desempeñan un papel relevante.

El concepto sociedad del conocimiento proporciona una comprensión de las transformaciones presentes de la modernidad, debido a que materializa las relaciones entre el desarrollo económico, la información y la educación, al tiempo que aporta a la toma de decisiones futuras desde el accionar político, por las posibilidades de participación e interacciones de los ciudadanos (Krüger, 2006). Desde esta postura, la formación laboral y académica del capital humano cobra relevancia para el crecimiento económico y el cambio social (Márquez Jiménez, 2017). En este sentido, los procesos de producción y divulgación científica, en la sociedad del conocimiento, se convierten en ejes de desarrollo e interacción, lo que genera una responsabilidad de la educación superior en la formación de la escritura académica en los estudiantes, orientada a incrementar su participación en comunidades científicas, mediada por la socialización y el intercambio de resultados de investigaciones y la producción reflexiva y crítica, desde los saberes disciplinares y multidisciplinares.

Por otra parte, los estudiantes de Iberoamérica reconocen la importancia de las competencias comunicativas en su formación como profesionales, pero expresan que el ingreso a la cultura académica universitaria se vuelve un desafío (Gavari Starkie y Tenca Sidotti, 2017; Zárate Fabián, 2017), en tanto son pocas las asignaturas que fortalecen o introducen a los estudiantes en estas nuevas dinámicas; y estas, a su vez, solo se focalizan en aspectos más lingüísticos de la lengua materna (Núñez Cortés y Moreno Núñez, 2017). Además, algunos profesores privilegian los productos, en vez del proceso mismo de la escritura (Fernando, 2018), lo que hace que se pierda una oportunidad didáctica para cualificar dichas competencias comunicativas.

Las condiciones actuales de la educación superior, como ampliación de la cobertura a diferentes poblaciones, internacionalización e investigación, exigen no solo un seguimiento y acompañamiento más personalizado del estudiante durante toda la formación, debido a que a la universidad llegan estudiantes con diferentes niveles de desempeño académico, culturas e intereses formativos (Álvarez Pérez et al., 2016), sino también fortalecer sus competencias comunicativas en las disciplinas (Bucur y Ban, 2019; Calle Álvarez, 2017), lo cual contribuiría con la cualificación de los aprendizajes orientados a lograr la formación integral, mejorar el rendimiento académico y garantizar su permanecía en la universidad.

Por otro lado, no solo en los estudiantes se han identificado dificultades en la producción de textos académicos; en los profesores también se han explicitado los retos que les implica la producción científica y el uso de las tecnologías digitales para la escritura colaborativa, la divulgación y la visibilización de los resultados de sus investigaciones, el valor de la escritura en espacios como los blogs y las redes sociales, los límites entre el espacio laboral y familiar (Barton y McCulloch, 2018), y la propiedad intelectual (Gainza, 2016).

La relación entre lectura, escritura y tecnologías digitales es una línea de investigación de interés vigente entre profesores e investigadores (Casanovas Catalá, 2016; Figueroa y Aillon, 2015; Rodríguez Málaga et al., 2019), buscando 
entender cómo utilizar las tecnologías para la enseñanza de la escritura; su potencial impacto en el desarrollo de los avances tecnológicos; la identificación de vacíos existentes en su enseñanza (Amante Negrete y Gómez Zermeño, 2017; Strobl et al., 2019); el análisis de las maneras en que los nuevos géneros, prácticas de colaboración y participación en la escritura afectan el aprendizaje (Magnifico et al., 2020), y la evaluación de las competencias escriturales (Fernando, 2018).

Las teorías sobre la escritura académica y sus consecuencias en decisiones prácticas asociadas a la estrategias universitarias para fortalecerlas, particularmente en el ámbito iberoamericano, aún requieren estudios que permitan obtener mayores comprensiones relacionadas con la cualificación de los procesos de alfabetización académica y digital en el campo de la formación profesional desde la escritura científica, así como la adquisición de habilidades para la interacción en plataformas digitales desde la autonomía y la autogestión del conocimiento.

Por ello, se diseñó un estudio de caso, con tres fases: la fase 1, la revisión documental, de la investigación desarrollada, analiza estudios asociados a la escritura en la educación superior, desde las cuales ampliar su horizonte de comprensión, y para establecer bases conceptuales orientadas al diseño de un centro de escritura digital (CED) universitario como espacio educativo y formativo, focalizado en el fortalecimiento de la escritura académica. Por su parte, la fase 2 se ocupa del diseño y pilotaje del CED y la fase 3 , de la consolidación y el análisis de la información.

En este sentido, el diseño y la operación de este espacio requieren, atendiendo a las perspectivas teóricas planteadas previamente, estudiar cuáles pueden ser los aportes de las investigaciones sobre escritura académica para la fundamentación teórica en la configuración de un CED universitario, pregunta de investigación que orientó la fase 1 y de la que da cuenta este artículo.
Son muchos los avances publicados sobre la alfabetización académica en aspectos como la enseñabilidad de la escritura en las disciplinas y el crecimiento profesional (Carlino, 2017), el uso de las tecnologías digitales como mediadoras del aprendizaje (Amante Negrete y Gómez Zermeño, 2017), la escritura académica como dispositivo cultural (Ortiz Casallas, 2015), su valor epistémico (Álvarez y Difabio de Anglat, 2018) y el uso de la escritura en los procesos de evaluación y mejoramiento académico (Fernando, 2018). Desde el horizonte de indagación de esta investigación, el reconocer estos avances aporta, al estudio sobre el diseño de un CED, comprensiones en la configuración de líneas de orientación de la escritura para los estudiantes y profesores, mecanismos de comunicación y divulgación en la comunidad universitaria, estrategias de sostenimiento y fundamentación teórica del CED.

Estudiar el funcionamiento de un CED en la educación superior parte de la fundamentación teórica, como base para su diseño y posterior experimentación empírica en la Universidad de Antioquia, orientado por el propósito general de contribuir a generar conocimiento sobre los procesos de aprendizaje y enseñanza de la escritura académica, las alternativas didácticas para la educación en un entorno virtual, y las posibilidades pedagógicas para el acceso y el acompañamiento de los estudiantes desde diferentes ubicaciones geográficas.

En la actualidad, la enseñanza y el aprendizaje de la escritura académica en la educación superior se encuentra mediada por la cultura digital. Esta situación ha implicado la confluencia de la alfabetización académica y la alfabetización digital en la formación de los profesionales. La enseñanza de la escritura académica analógica es insuficiente, por las posibilidades de la comunicación y el aprendizaje multimodal que emergen en las producciones escritas contemporáneas, retos que exigen estudiar, diseñar y experimentar metodologías de trabajo alternativas, campo en el que se inscribe la presente investigación. 
De la convergencia entre la alfabetización académica y la digital emergen los centros de escritura en línea, los cuales, dependiendo de la región geográfica donde funcionan, han asumido otros nombres, como "laboratorio de escritura virtual", "laboratorio de escritura en línea", "centro de multialfabetizaciones" y "centro de escritura digital". Sin embargo, todos mantienen el principio de la formación de la escritura en los usuarios, desde la perspectiva de los contextos unimodales y multimodales, debido a que las relaciones entre enseñanza, aprendizaje y cultura digital implican que los estudiantes comprendan las lógicas digitales y analógicas para la producción textual.

Finalmente, resulta conveniente precisar que las decisiones técnicas dependerán no solo de los recursos económicos y de personal disponibles, sino esencialmente del diseño académico (disciplinar, pedagógico y didáctico) y los propósitos formativos bajo los cuales se fundamenten. En síntesis, la revisión documental, como parte de la fase 1 del estudio, pretende aportar a las comprensiones sobre los centros de escritura en la educación superior (Clarence y Dison, 2017), consolidar los argumentos orientados a trascender el enfoque remedial que ha predominado en la mayoría de iniciativas universitarias sobre los procesos de lectura y escritura, para reivindicar, a través de ellas, la formación humana, profesional y científica. De igual manera, se busca superar, en algunas iniciativas universitarias, la concepción de la escritura como técnica, cuya destreza mantiene los índices de permanencia y retención estudiantil, entendiéndola como posibilidad para la formación, la creación de espacios de participación y el fortalecimiento de la autonomía cognitiva.

\section{Método}

El método utilizado consistió en una revisión documental sistemática de artículos asociadas a la escritura académica. La base documental está constituida por un corpus final de 58 textos, producto del análisis preliminar obtenido de un repositorio inicial de 100 producciones académicas publicadas entre los años 2014 y 2020, incluidas en las bases de datos Dialnet, Ebsco, Publindex, Scopus o Web of Science.

El corpus fue seleccionado atendiendo, además, a criterios de orden teórico, utilizando la intersección entre los descriptores "centro de escritura", "escritura académica”, "alfabetización académica”, "escritura científica" y "educación superior", con su equivalente en lengua inglesa; y de orden metodológico, priorizando estudios de naturaleza empírica ( $83 \%$ de los estudios incluidos).

La selección final respondió a criterios de calidad y pertinencia para el propósito general del análisis, su relación directa en el ámbito de la educación superior universitaria; la relación teórica (no instrumental) entre educación y tecnología digital; y la población estudiantil universitaria regular (no excepcional). Además, su elección refleja una postura investigativa y global de la comprensión transversal de los fundamentos teóricos y prácticos de la alfabetización académica, lo que aporta, desde la teoría y la didáctica, al diseño y funcionamiento de un CED.

Para la consolidación de la información, como instrumento fue diseñada una ficha documental, integrada por dos apartados: uno para registrar los metadatos del texto objeto de análisis y el otro para su estudio y categorización. Este instrumento tuvo un desarrollo instruccional para su utilización digital.

El corpus fue analizado mediante el lenguaje de programación Python $3.7^{\circ}$, utilizando principalmente la librería de lenguas naturales (Natural Language Toolkit) y técnicas de análisis del discurso, siguiendo un proceso de extracción, el cual conlleva la preparación de las unidades de análisis; el preprocesamiento, que involucra la unificación de caracteres, puntuación, corrección ortográfica y normalización de palabras (Korenius et al., 2004); y, finalmente, el análisis de texto, utilizando criterios de polarización, recurrencia y estructura de bigramas. 
Esta perspectiva analítica, atendiendo a los términos de Graham (2008), implica la identificación de líneas de estudio, convergencias y divergencias entre las investigaciones objeto de análisis, presentadas bajo tres tópicos para la discusión: el primero explicita algunas características geográfico-temporales emergentes derivadas del corpus documental examinado; el segundo contrasta las líneas predominantes para el estudio de la escritura académica, y el tercero determina los componentes pedagógico-didácticos, de formación disciplinar, técnicos y relacionados con la comunidad virtual de aprendizaje, involucrados en el fortalecimiento de la escritura académica, base para el diseño del CED.

\section{Resultados y discusión}

En el análisis del corpus se identificaron aspectos espaciotemporales, desde el origen de los estudios en 24 países. Es preciso aclarar que este componente bibliométrico no hizo parte de los propósitos iniciales de la revisión documental; por tanto, no fue considerado un criterio de búsqueda y selección de la información. Sin embargo, el análisis de estos metadatos aporta a la comprensión de las dinámicas, en diferentes territorios, sobre la escritura académica y el nivel de desarrollo para el caso iberoamericano, y particularmente para el contexto académico en el cual se desarrolla el estudio aquí propuesto.

Los resultados obtenidos muestran, por un lado, las líneas de investigación predominantes de la escritura académica y, por otro, los desarrollos teóricos y prácticos de los componentes pedagógicos-didácticos, técnicos, de formación disciplinary asociados a comunidades virtuales de aprendizaje, para su comprensión y posible implementación en una propuesta formativa como es un CED para la educación superior desde las realidades y necesidades institucionales.

\section{Características geográfico-temporales emergentes de las investigaciones y prácticas}

La mayoría de los textos seleccionados (82,8 \%) son artículos de investigación; un menor número, artículos de revisión $(8,6 \%)$ o experiencias $(6,9 \%)$ y, finalmente, capítulos en libros (1,7\%), publicados básicamente en el periodo comprendido entre los años 2017 y 2019 (78 \% del corpus).

Como se muestra en la Figura 1, los textos seleccionados corresponden a publicaciones editadas en 15 países. En contraste, en el origen de su contenido (nacionalidad de los investigadores, financiación, población o instituciones participantes), se registra la vinculación de 24 países.

Los resultados muestran que los estudios sobre escritura académica en la universidad representan un interés para la sociedad científica global, por sus implicaciones en la vida académica, profesional y cultural. Colombia, de manera particular, viene aumentando su producción científica sobre objetos de estudio asociados a la calidad de los programas de escritura (Maldonado, 2017; Moreno Mosquera, 2019), las estrategias de enseñanza y aprendizaje en entornos digitales (López Gil, 2016), las tutorías académicas (Calle Arango, 2019; Molina Natera,

Figura 1 Clasificación del corpus de textos seleccionados de acuerdo con el país de la publicación y el país origen de su contenido

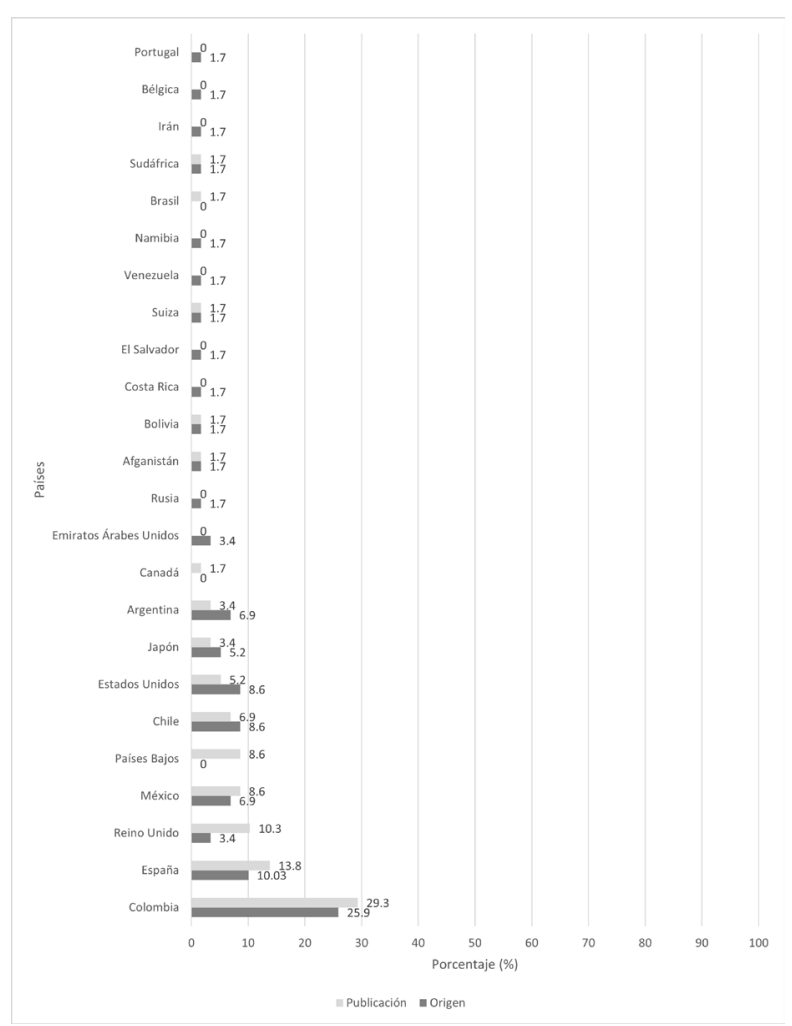


2017) y la implementación de centros de escritura (Chaverra, 2019; Sito et al., 2019). La investigación originada en su territorio (25,9\%) es divulgada entre la comunidad científica mediante publicaciones dirigidas bajo sus propios académicos (29,3\%). Es este un dato relevante, en la medida en que no siempre el país donde se origina la investigación cuenta con publicaciones científicas para su divulgación nacional e internacional.

Si bien, como se evidencia en la Figura 1, los países europeos sobresalen como productores y receptores de la productividad científica, con una amplia experiencia en los procesos de alfabetización académica (Bucur y Ban, 2019; Carlino, 2013; Casanovas Catalá et al., 2019; Fernández Jiménez et al., 2017; Gavari Starkie y Tenca Sidotti, 2017), resulta relevante la consolidación que al respecto vienen teniendo las investigaciones iberoamericanas, particularmente en los estudios relacionados con los centros de escritura (Calle Arango, 2019; Casanovas Catalá et al., 2019; Hermosillo y Verdín, 2018; Molina-Natera y López-Gil, 2020). En dichos estudios no solo se da cuenta de la evolución y el posicionamiento de estos centros de escritura en las instituciones de educación superior, en el sentido de construir una identidad y en la formación de los estudiantes, sino también la cualificación de sus servicios, como, por ejemplo, el sentido pedagógico-didáctico de la tutorías, el trabajo colaborativo como estrategia para mejorar la escritura académica, o el rol de las tecnologías digitales en los centros de

Figura 2 Descriptores derivados de palabras clave. Recurrencia de los descriptores presentes en el corpus analizado

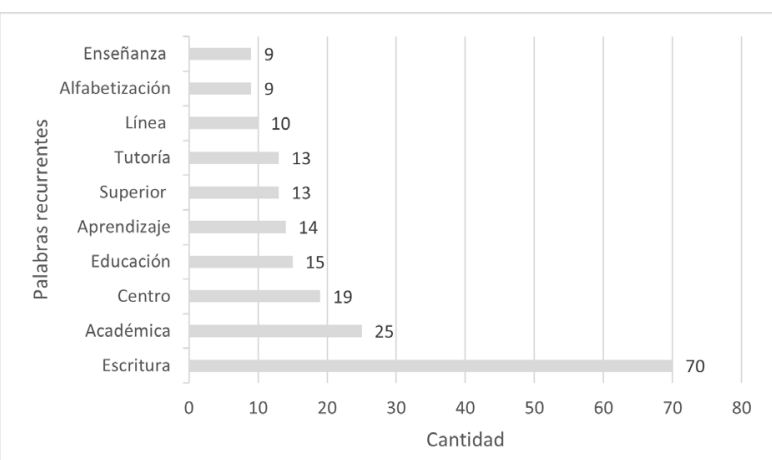

escritura como posibilidad para promover y enriquecer la producción textual.

\section{Líneas predominantes para el estudio de la escritura académica}

De acuerdo con la identificación de patrones y relaciones (minería de datos) y el análisis de contenido de la revisión documental efectuada, se evidencian dos líneas predominantes y relacionadas con el estudio de la escritura académica, las cuales aportan a la discusión epistemológica y empírica, en particular en el ámbito iberoamericano.

Las Figuras 2 y 3, erigidas a partir de las palabras clave y los resúmenes de la información documental objeto de análisis, muestran, en primer lugar, la consolidación de los centros de escritura a los cuales se vinculan el uso de las tecnologías digitales, lo que se evidencia en la recurrencia de palabras como "centro", "escritura" y "línea" en el corpus analizado (Calle Álvarez, 2019; Salisbury, 2018; Stella y Corry, 2016; Strobl et al., 2019); en segundo lugar, la comprensión de la alfabetización académica, lo que se evidencia en el bigrama de la Figura 3 asociado a las posibilidades de la enseñabilidad y el aprendizaje de la escritura y la lectura en la educación superior (Carlino, 2018; Molina-Natera y López-Gil, 2020; Núñez Cortés y Moreno Núñez, 2017). Ambas líneas evidencian el interés que mantienen las universidades en promover y fortalecer la escritura académica en los estudiantes, por su importancia para el aprendizaje y la divulgación de los saberes disciplinares.

Figura 3 Bigrama derivado de resúmenes. Recurrencia de la relación binaria entre los descriptores presentes en el corpus analizado

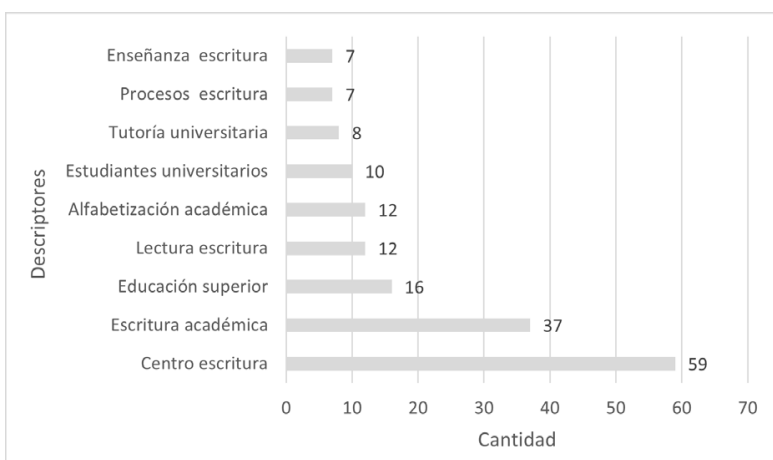


En la primera línea de estudio (Figura 4), los centros de escritura son entendidos como estrategias institucionales para la formación de mejores escritores, los cuales tuvieron su origen en Estados Unidos de América, y actualmente se han identificado centros de escritura en 66 países, Colombia entre ellos (Calle Arango,2019; GavariStarkieyTencaSidotti, 2017; Molina Natera, 2016; Tiruchittampalam et al., 2018). Su diseño, operación y actualización responde a los retos e intereses particulares de las universidades (Thompson, 2014). Su objetivo formativo está centrado en el aprendizaje y el desarrollo de las habilidades de escritura en los estudiantes (Al Murshidi y Al Abd, 2014; Peña Portero y Santamaría Martínez, 2020). En la actualidad, existe la Asociación Internacional de Centros de Escritura, integrada por diferentes asociaciones regionales.

Un centro de escritura ofrece diferentes servicios para la orientación de la escritura académica de los estudiantes, como complemento a los procesos de enseñanza de la escritura que se brinda desde las disciplinas (Calle Arango et al., 2017). Su consolidación responde a los retos actuales de la educación superior en modalidad virtual, en términos tecnológicos y pedagógicos; para ello, combinan servicios presenciales y virtuales, o funcionan exclusivamente en línea. Así, las instituciones de educación superior buscan responder a las demandas espaciales y temporales de los estudiantes, a la cobertura, a los ritmos y estilos de aprendizaje, y para ello utilizan, además, recursos como videos o guías para contribuir con sus necesidades de asesoría asociadas a la escritura (Fernando, 2018; Thompson, 2014).

Las modalidades virtuales e híbridos para ofrecer los servicios de los centros de escritura evidencian las potencialidades de las tecnologías digitales como herramientas de ayuda dentro del proceso educativo, ya que posibilitan llegar a un número mayor de personas y permiten la optimización de nuevas posibilidades comunicativas y formativas (Martínez Clares et al., 2016). Como sostienen Crowley-Watson (2019), se pueden combinar los espacios presenciales y virtuales para promover la asistencia a los centros de escritura, por ejemplo, mediante la escritura guiada apoyada por las redes sociales. Sin embargo, su incorporación implica

Figura 4 Representación visual de las líneas de estudios sobre centros de escritura a partir de conceptos clave

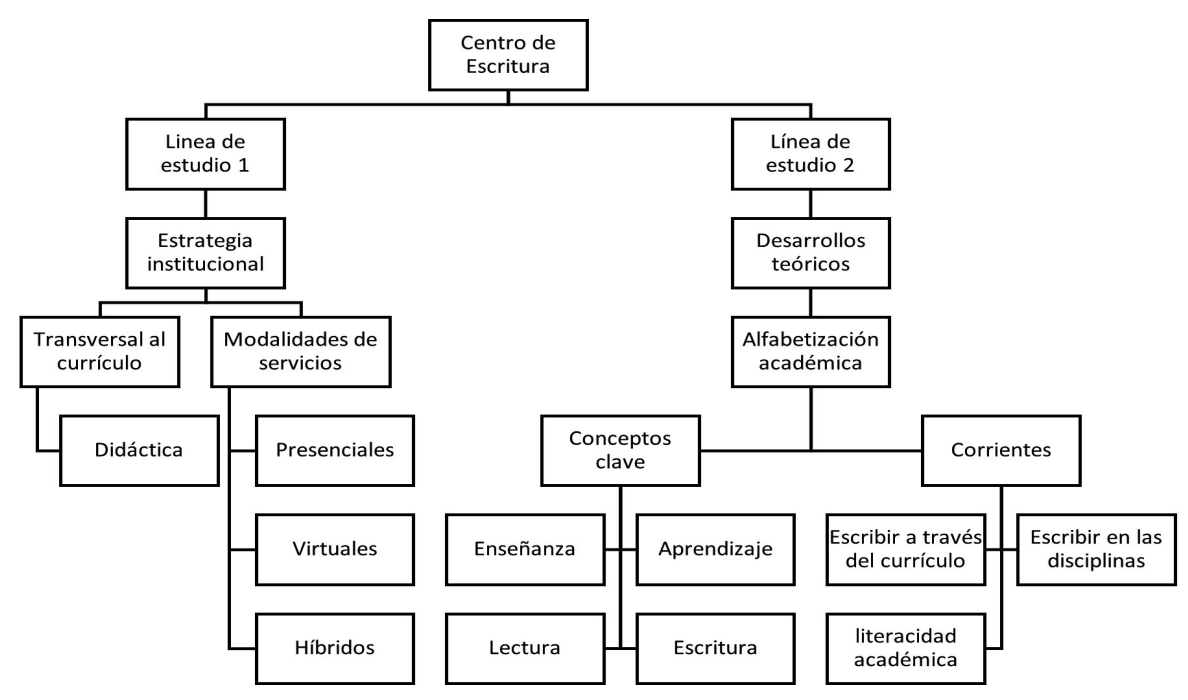


otras comprensiones de los procesos de enseñanza y aprendizaje (Fernández Jiménez et al., 2017), que no se limitan a las herramientas de acceso y conectividad.

En la segunda línea de estudio focalizada en la comprensión de la alfabetización académica (Carlino, 2013; Molina-Natera y López-Gil, 2020), se identifican tres corrientes que tienen una tradición investigativa. En los estudios de Estados Unidos se reconocen los movimientos "escribir a través del currículo" (WAC) y "escribir en las disciplinas" (WID); también están las investigaciones británicas y australianas sobre la "alfabetización académica" (Carlino, 2017; Maldonado, 2017; Núñez Cortés y Moreno Núñez, 2017), y en Latinoamérica, una línea sociocultural denominada "literacidad académica" (Sito et al., 2019). En el contexto latinoamericano resulta necesario adelantar investigaciones que permitan precisar el espacio de la alfabetización académica en la formación disciplinar (Navarro et al., 2016). En todas ellas, converge el interés por proponer estrategias, metodologías o materiales bajo los cuales orientar procesos de enseñanza, y particularmente comprender y estimular el aprendizaje de los estudiantes.

Las dos líneas de investigación que emergieron del análisis (centros de escritura vinculados al uso de las tecnologías digitales y comprensión de la alfabetización académica) son susceptibles de relación entre sí, en tanto ambas fortalecen los desarrollos teóricos y estudios empíricos sobre la escritura académica y la incorporación de las tecnologías digitales a la educación superior, convergencia incipientemente denominada "posalfabetización” (Mora y Golovátina-Mora, 2020). Los usuarios de un CED requieren habilidades tecnológicas asociadas al uso del entorno, además de desarrollar competencias funcionales y críticas para la búsqueda, la selección, el análisis y el uso de la información (Polizzi, 2020), y asumir la escritura multimodal para la construcción y la divulgación del conocimiento (Vu et al., 2019). Sin embargo, es precisamente en la segunda línea en la que la escritura académica cobra relevancia, por el reconocimiento de las posibilidades de enseñabilidad y aprendizaje en la educación superior y, por tanto, constituye el referente principal desde el cual vincular el diseño del CED y, a su vez, retornar los resultados que este pueda generar en la fase 2 (estudio empírico).

\section{Componentes pedagógico-didáctico, de formación disciplinar y técnico involucrados en el fortalecimiento de la escritura académica}

El análisis parte del esquema categorial que se muestra en la Figura 5, adaptado a partir del propuesto por Chaverra (2019). Es una matriz previa,

Figura 5 Sistema de categorías aplicado a la revisión documental
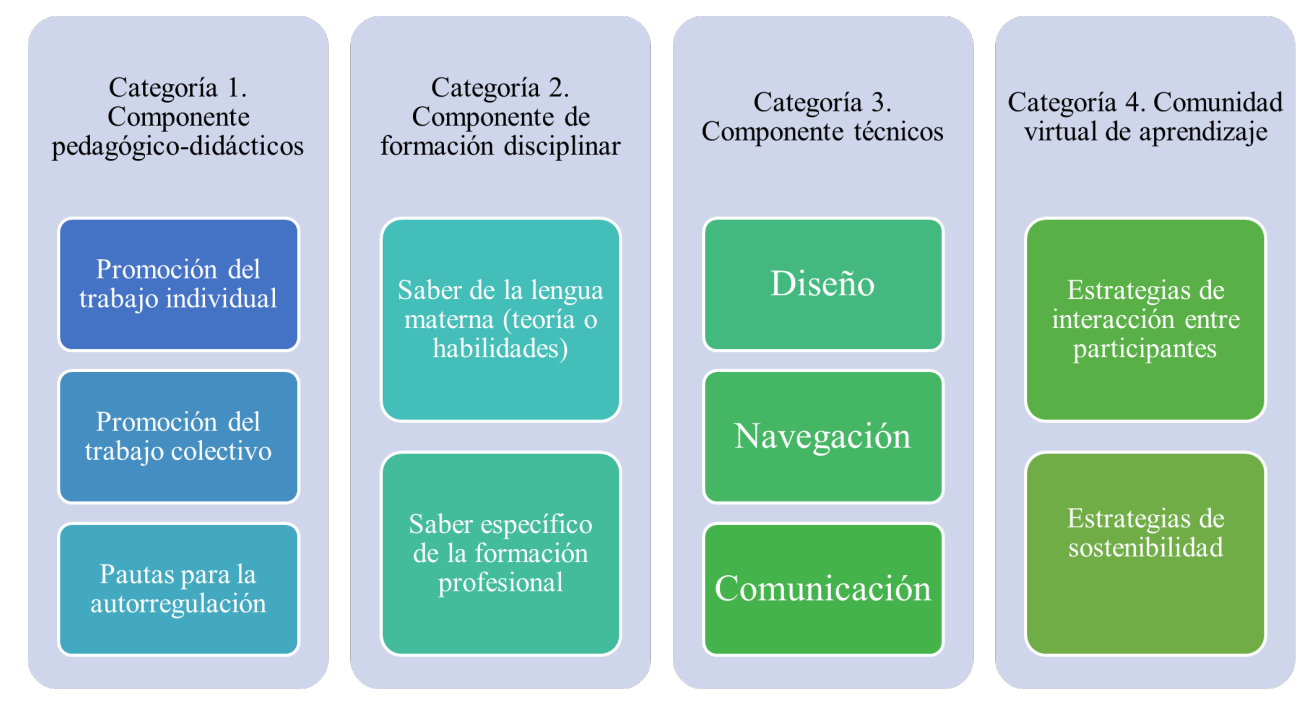
mediante la cual se diseñó el instrumento inicial, completado con elementos emergentes derivados de su aplicación.

- Componente pedagógico-didáctico. En este se concentran los principales hallazgos y aportes de las investigaciones revisadas (véase Figura 6). Este componente cobra sentido porque aporta a la comprensión social y formativa de la escritura académica en la educación superior, superando la postura instrumental. Además, reafirma su importancia para el aprendizaje, la conformación de comunidades académicas y la participación en la sociedad del conocimiento.

Los estudios refieren, en gran proporción, análisis relacionados con las subcategorías "promoción del trabajo individual - atender la singularidad" $(43,1 \%)$ y "promoción del trabajo colectivo aprender con el otro" (29,3\%), lo cual indica que en ellos se promueve tanto la atención a la singularidad como la construcción conjunta.

Los modelos de educación superior actual conciben al estudiante como sujeto central del aprendizaje, que adquiere de manera autónoma competencias profesionales y personales (Lobato Fraile y Guerra Bilbao, 2016), apoyado, además, en las tecnologías multimodales (Amante Negrete y Gómez Zermeño, 2017), lo que favorece la enseñanza de la escritura desde una perspectiva comunicativa y creadora (Clerici, 2017). Lo anterior se corresponde con la postura de algunos centros de escritura, que promueve la apropiación de manera autónoma de las habilidades de lectura y escritura académica, que le permita al estudiante un aprendizaje a lo largo de la vida, y su aplicación en diferentes espacios sociales y científicos (Limpo y Alves, 2018; Molina-Natera y LópezGil, 2020).

Respecto a la subcategoría "generación de pautas para la autorregulación-incentivar la autorrevisión", se promueve el fortalecimiento de habilidades del pensamiento y del lenguaje. Así, uno de los pilares de los centros de escritura es la formación de mejores escritores académicos; por ello, los tutores establecen un diálogo con los usuarios sobre sus habilidades y no sobre los errores gramaticales del texto. Desde esta perspectiva, el centro de escritura no es un lugar de corrección de textos (Molina Natera, 2016; Thompson, 2014), sino un espacio en el cual pueden tomar conciencia sobre los diferentes elementos lingüísticos y discursivos que caracterizan un texto "bien escrito".

Igualmente, desde las relaciones entre las categorías "promoción del trabajo colectivo-aprender con el otro", la "promoción del trabajo individual - atender la singularidad"

Figura 6 Resultados categoría componente pedagógico-didáctico.

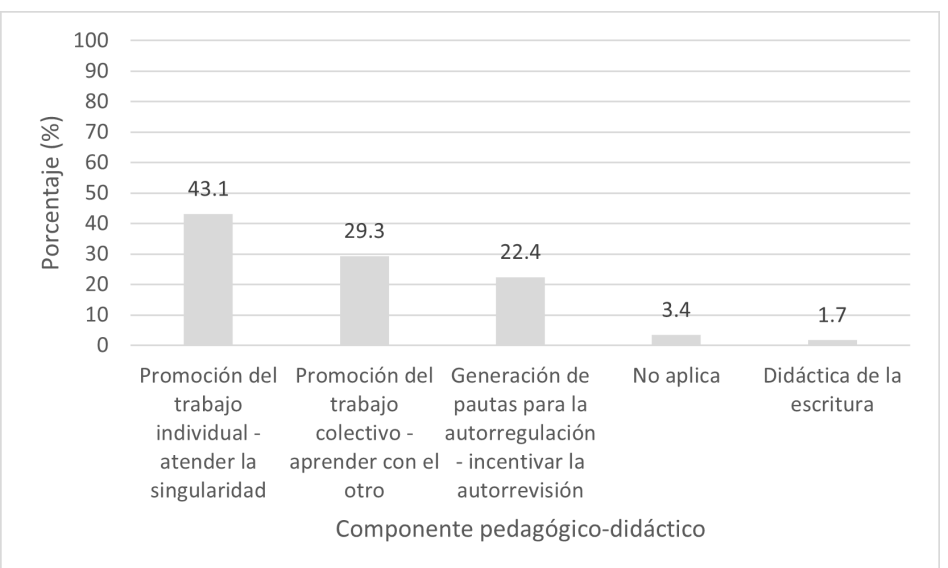

Nota: El porcentaje obtenido en la barra "No aplica" alude a la cantidad de estudios que no reportaron aspectos asociados a este componente. 
y la "generación de pautas para la autorregulación-incentivar la autorrevisión”, emerge la tutoría universitaria y académica.

La tutoría se puede considerar un factor de calidad en la educación superior, si se concibe como un proceso de acompañamiento integral del estudiante. No consiste en el uso de alguna herramienta tecnológica; implica, más bien, la integración de diversos recursos para llevar a cabo un proceso de orientación, comunicación e intercambio de información (Lobato Fraile y Guerra Bilbao, 2016; Martínez Clares et al., 2016).

La tutoría es un espacio adecuado para desarrollar los procesos cognitivos de los estudiantes (Calle Arango, 2019), promover el aprendizaje colaborativo (Molina Natera, 2017), potenciar las competencias transversales en la educación superior (Álvarez Pérez et al., 2016) e inducir procesos de reflexión acerca de la propia escritura en el estudiante (Munje et al., 2018).

La tutoría universitaria en general o la académica en específico, se entienden como estrategias relevantes y transversales del proceso formativo (Álvarez Pérez et al., 2016). Ocupan un lugar relevante en los centros de escritura. Dependiendo de su organización, esta puede ser orientada por profesores, estudiantes de posgrado o pares académicos. Esta última se caracteriza por el trabajo entre iguales (Bleakney, 2019; Broekhoff, 2014; Chois-Lenis et al., 2017; Latifi et al., 2019; Molina Natera, 2019; Munje et al., 2018). En ella se privilegia el proceso de escritura y la lectura activa conducente a la construcción de argumentos (Calle Arango et al., 2017; Fernando, 2018), el aprendizaje colaborativo y la construcción social del conocimiento (Molina Natera, 2017; Sito et al., 2019).

Con respecto a la subcategoría "didáctica de la escritura", esta se presenta como una posibilidad para el acompañamiento del proceso de escritura, el cual requiere de gran esfuerzo cognitivo por parte del escritor; por ello, la forma en que se acompaña, por parte del profesor o tutor, desde el momento de concebirla, puede determinar la calidad, la profundidad y estilo de la misma (Limpo y Alves, 2018). Igualmente, el trabajo colaborativo desde los centros de escritura puede aportar a la enseñanza y el aprendizaje de la escritura a través del currículo (López-Gil y Molina Natera, 2018) y la conformación de comunidades de aprendizaje (Figueroa y Aillon, 2015). Lo anterior se convierte en un desafío para los profesores, debido a las dificultades que presentan los estudiantes para negociar y consensuar, lo que evidencia la necesidad de fomentar la escritura colaborativa, el diálogo, la lectura del otro (Clerici, 2017) y los aspectos sociales en la escritura en espacios virtuales (Magnifico et al., 2019).

En el componente pedagógico-didáctico de la producción académica revisada, prima la reivindicación de atender y respetar la singularidad de los sujetos y, por tanto, se reconoce la importancia del trabajo individual, sin desconocer las bondades del trabajo colectivo como una oportunidad para poner en acción la inteligencia colectiva, en pro de resolver los problemas que implica el acto de escribir. Una perspectiva que se traduce en la participación activa, conversación y realimentación formativa en el desarrollo de los servicios promovidos desde un centro de escritura.

- Componente de formación disciplinar. En las subcategorías que integran este componente (véase Figura 7), se hace notorio el énfasis de las investigaciones y prácticas en la promoción y el fortalecimiento del saber de la lengua materna como objeto de estudio, desde la teoría y la práctica, para el desempeño de los estudiantes en la vida académica (69,0\%); en menor proporción, la relación de la escritura con el saber específico de la formación profesional (10,3 \%). Una relación que, desde la línea de investigación que estudia la escritura a través del currículo, conviene fortalecer. Los centros de escritura se constituyen en una alternativa para ello. 
Figura 7 Resultados categoría componente de formación disciplinar.

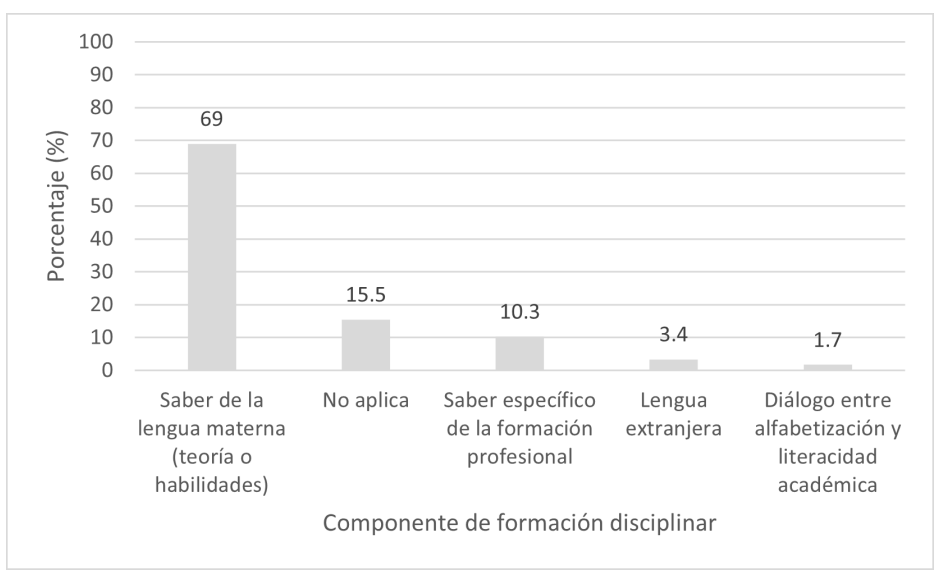

Nota: El porcentaje obtenido en la barra "No aplica" alude a la cantidad de estudios que no reportaron aspectos asociados a este componente.

En algunos programas académicos, las competencias comunicativas, entre ellas la escritura, son consideradas como habilidades blandas, lo que conduce a que los estudiantes no les den la importancia que deberían tener para el aprendizaje disciplinar (Bucur y Ban, 2019). Otra razón es que son pocos los profesores que utilizan la lectura y la escritura en sus áreas para la discusión e intercambio de saberes (Carlino, 2018). Sin embargo, su dominio supera la transcripción de la lengua y promueve la participación de los estudiantes en la sociedad (Calle Álvarez, 2017; Hermosillo y Verdín, 2018).

Desde las relaciones teóricas y prácticas de las subcategorías "saber de la lengua" y "saber específico”, se identifica que la lectura académica en la universidad no es un tema que interese exclusivamente a los estudiosos del lenguaje; requiere una reflexión y participación de los profesores de las disciplinas y la articulación con la escritura a través del currículo (Moreno Mosquera, 2019).

La lectura y la escritura se convierten en herramientas potentes para desarrollar el pensamiento sobre los objetos de las disciplinas y mecanismos para la discusión con otros (Carlino, 2017). Además, cuando un estudiante ingresa a la universidad, inicia la formación en un saber específico que requiere el análisis y la reflexión de los contenidos, donde la escritura se convierte en un apoyo para el desempeño académico (Ballén Vargas y Herrera González, 2017). Esta situación provoca compromisos en la enseñanza desde el plan retórico, el conocimiento disciplinar y los aspectos idiomáticos (Clarence y McKenna, 2017; Romero González y Álvarez Álvarez, 2019).

Las conclusiones del estudio realizado por Kozolup et al. (2020) con estudiantes universitarios de Europa del este, si bien no son novedosas, sí son recurrentes y convergentes con los resultados de la revisión realizada y los aportes de la investigación al campo de estudios. Estas conclusiones señalan que los estudiantes necesitan más enseñanza en escritura académica, mayor interacción con los géneros propios de su campo disciplinar tanto en lengua materna como extranjera, y más conciencia del papel que cumple el lenguaje y la comunicación en su desarrollo personal, así como del potencial educativo de la escritura.

Por otra parte, desde la subcategoría "lenguas extranjeras", en sus inicios los centros de escritura estaban centrados en el fortalecimiento de la escritura académica en lengua materna; pero, con una sociedad global, se ampliaron a otras lenguas (Tiruchittampalam et al., 2018). 
Korotkina (2017) recomienda un enfoque para incorporar la escritura académica mediante la creación de redes entre investigadores, académicos y maestros, y el enfoque bilingüe para su enseñanza, que puede ayudar a introducir las mejores prácticas internacionales de manera flexible y eficiente. Esta postura coincide con la de Elejalde Gómez y Ferreira Cabrera (2018), en cuanto al fomento de la autonomía y la participación del estudiante en su proceso de aprendizaje, a partir del reconocimiento del error en la aplicación de las habilidades comunicativas en la lengua extranjera.

Los hallazgos del estudio de Okuda (2020) causan un contraste parcial con los análisis aquí reportados, en tanto en esta categoría (componente de formación disciplinar) resulta como punto convergente el determinante papel que cumplen los tutores como especialistas del lenguaje para orientar los procesos de escritura en el CED; sin embargo, dicho perfil no solo debe aludir a su competencia lingüística y comunicativa, sino también a su competencia científica, en tanto son especialistas en su campo de conocimiento. Bajo esta perspectiva, el acompañamiento para escribir no es exclusivamente técnico, postura comprensible en el estudio de Okuda, al asumir la teoría de la gestión del lenguaje (Language Management Theory) como fundamento de la escritura desde el cual orientar las tutorías en el centro de escritura de la universidad nipona donde desarrolla sus estudios.

- Componente técnico. Como se identifica en la Figura 8, el $63 \%$ de las investigaciones analizadas presenta aportes de carácter técnico, referidas en su mayoría (31\%) a elementos de comunicación, que aluden a las posibilidades de intercambio de información entre los usuarios y son requeridos en la utilización de medios para el acompañamiento de las prácticas de escritura en los ced. Los estudiantes tienen acceso a una variedad de recursos digitales sobre diversos saberes que van más allá de las aulas de clases; sin embargo, eso no quiere decir que su uso sea efectivo y aporte a los aprendizajes de las disciplinas. Por ello, es importante comprender la dimensión técnica de los ced desde la perspectiva formativa que los caracteriza.

Desde las subcategorías de "diseño" y "navegación", los centros de escritura han diversificado sus estructuras y servicios con el auge de las tecnologías digitales (Calle Álvarez, 2019). Se entiende acá el "diseño" como la planificación

Figura 8 Categoría componente técnico.

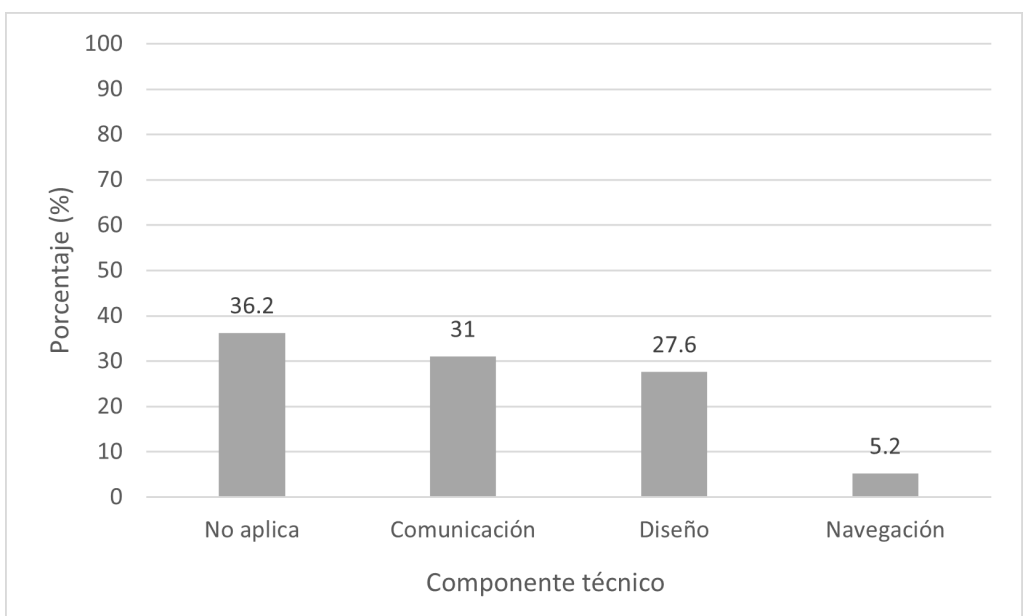

Nota: El porcentaje obtenido en la barra "No aplica" alude a la cantidad de estudios que no reportaron aspectos asociados a este componente. 
de los elementos del entorno del CED para su presentación visual, mientas que la "navegación” hace referencia a las formas de acceso a los diferentes elementos del CED y que favorecen la ubicación del usuario en los servicios.

El diseño de una página web para la comunicación y la divulgación de los servicios requiere instrucciones precisas en sus alcances para el acceso y uso, debido a que ello es una oportunidad para que los estudiantes ingresen y comprendan los objetivos del CED o, por el contrario, puede ser un obstáculo (Al Murshidi y Al Abd, 2014).

En el diseño es importante considerar el componente técnico desde donde se va a estructurar y operar el CED (Calle Álvarez, 2019). Los soportes virtuales de un centro de escritura dependen de los recursos tecnológicos y financieros con que cuente; por ejemplo, algunos funcionan con recursos gratuitos, y otros, con plataformas LMS (Learning Management System o Sistema para la Gestión del Aprendizaje) pagas (Thompson, 2014). Sin embargo, un sistema de gestión de curso no es suficiente para aprender, y un problema abordado en comunidad puede inducir más a la participación y el aprendizaje por parte de los estudiantes (Salisbury, 2018).

Igualmente, desde la subcategoría de "comunicación”, una forma de divulgar los servicios del CED es por medio del correo electrónico, enviando periódicamente información (Al Murshidi y Al Abd, 2014; Thompson, 2014). Asimismo, se pueden aprovechar las redes sociales para intercambiar información sobre escritura académica, motivar la participación de los servicios del centro de escritura y aclarar dudas de los usuarios (Crowley-Watson, 2019), o crear portafolios digitales para el seguimiento del desempeño de la escritura (Iwasaki et al., 2019). Además, las tecnologías digitales permiten el acceso a documentos compartidos, materiales virtuales para la enseñanza y videoconferencias, en cualquier momento y lugar, lo que implica un reto para diferenciar el tiempo del trabajo y no trabajo (Barton y McCulloch, 2018).

El componente técnico, en las estrategias para el acompañamiento de la escritura académica mediada por tecnologías digitales, viene adaptándose y diversificándose según evolucionan las posibilidades de la tecnología educativa. Así, existen sistemas de tutorías inteligentes que apoyan los procesos de escritura, ya sea en aspectos mecánicos o de orden superior, los cuales pueden ser un apoyo al profesorado durante la enseñanza; sin embargo, los estudios existentes no han demostrado su total eficacia (Rodríguez Málaga et al., 2019). Igualmente, el soporte automatizado para revisar el nivel micro de los entornos virtuales está bien desarrollado, mientras que las herramientas para apoyar las estrategias de escritura son infrecuentes (Strobl et al., 2019). Además, el profesor/tutor desempeña un rol importante en el proceso de aprendizaje, por cuanto posibilita el desarrollo de la postura crítica y el intercambio reflexivo en la adquisición de las competencias comunicativas en la educación superior (Álvarez Pérez et al., 2016). Asimismo, la realimentación experta aporta a la calidad de la escritura (Álvarez y Difabio de Anglat, 2018).

En la virtualidad, la mediación didáctica es fundamental en la calidad de la escritura, en la medida en que es lo que permite superar una visión instrumental del componente técnico aplicado en los procesos de enseñanza. De esta manera, las herramientas digitales están en función de la búsqueda de información, planeación de la escritura, producción y revisión de los textos, hasta la divulgación de los mismos (Casanovas Catalá, 2016; Sánchez Ceballos, 2014; Stella y Corry, 2016).

- Componente comunidad virtual de aprendizaje. Como se observa en la Figura 9, con respecto a esta categoría, se destaca el "no aplica" 
(55,2\%), lo que se explica debido a que la búsqueda documental no estuvo centrada en ella. Sin embargo, sí se alcanzan a identificar dos características con potencial institucional para consolidar una comunidad virtual de aprendizaje, centrada en la promoción de la escritura académica: las "estrategias de interacción entre participantes" (37,9\%) y las "estrategias de sostenibilidad" (6,9\%).

Respecto a la subcategoría "estrategias de interacción entre participantes”, la inclusión de las tecnologías digitales en las estrategias de enseñanza y aprendizaje en la educación superior permite incrementar y hacer flexible la interacción estudiante-docente y estudianteestudiante, así como combinar diversos recursos multimedia y proveer de oportunidades para el aprendizaje colaborativo, entre otras cosas, a un costo relativamente bajo en términos de tecnología (Figueroa y Aillon, 2015; Márquez Guzmán y Gómez Zermeño, 2018).

Por otra parte, el uso de las tecnologías digitales para la escritura posibilita la producción con el otro y para otros, lo que facilita la divulgación de los textos (Clerici, 2017). Por ello, la comunicación en las comunidades virtuales está mediada por la lectura, la escritura y la oralidad (Elejalde Gómez y Ferreira Cabrera, 2018).
En la subcategoría "Estrategias de sostenibilidad", se evidenció que en los estudios sobre centros de escritura se identifica que un problema para el sostenimiento es la relación entre la financiación y los beneficios para la universidad. Por ello, el diseñar y operar un centro de escritura requiere comprensión de la gestión administrativa dentro de las universidades, para la consecución de los recursos físicos y financieros necesarios (Broekhoff, 2014; Thompson, 2014). Se precisa, entonces, generar una comunicación directa con los administradores y visibilizar las acciones desarrolladas desde el centro de escritura para las comunidades educativas. Igualmente, cada centro construye una gestión administrativa propia, dependiendo de las relaciones organizacionales de la universidad de la que hace parte (Calle Álvarez, 2017).

La gestión del centro de escritura implica retos en la formación del coordinador, la planeación de la prestación de los servicios en la universidad y la evaluación de la gestión y la calidad académica (Molina-Natera y López-Gil, 2020).

La perspectiva transversal de los componentes pedagógico-didáctico, técnico y comunidad virtual de aprendizaje evidencia que los nuevos enfoques formativos que demandan otros métodos de enseñar y formas de aprender, hacen necesarios cambios

Figura 9 Resultados componente comunidad virtual de aprendizaje

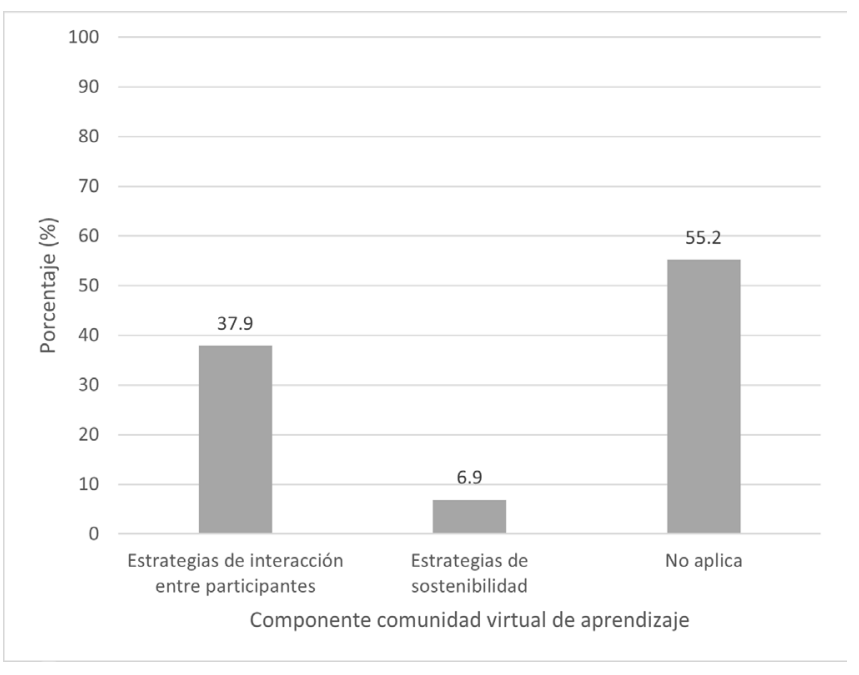


en las concepciones de las comunidades de aprendizaje. Atender a la diversidad y concebir la tutoría como un proceso de acompañamiento no solo del proceso de aprendizaje, sino también para el desarrollo integral de los estudiantes, son maneras de afrontar estos cambios de enfoque.

Las tecnologías digitales aportan al desarrollo de estos nuevos enfoques, siendo una herramienta de ayuda dentro del proceso educativo y orientador, ya que posibilitan llegar a un número mayor de personas y permiten la optimización de nuevas modalidades comunicativas y formativas (Martínez Clares et al., 2016).

En los centros de escritura se promueve la tutoría como complementaria de la función docente en sus distintas formas (personal, grupal y con apoyo virtual). De acuerdo con estudios realizados sobre la percepción de las tutorías virtuales, se evidencia que es la más empleada, pero la menos valorada por los estudiantes. Estos resultados invitan a pensar que la tutoría virtual debe aprovechar mejor todas las posibilidades de las tecnologías digitales, como herramientas que promuevan el desarrollo integral de los estudiantes, siempre que se optimicen y rentabilicen las plataformas creadas al efecto, y dejar de considerarla como una acción puntual y reactiva (Martínez Clares et al., 2016).

En los términos de Kruse y Rapp (2019), la ubicuidad, la temporalidad, la conectividad y la interactividad son algunas dimensiones que han marcado diferencias cuando la escritura es asociada a las tecnologías digitales respecto de la escritura análoga o manuscrita originada en la imprenta de Gutenberg. Dichos aspectos guardan relación con algunos de los postulados formulados por De Kerchov (1999), Jewitt y Kress (2008) y Sharples (1999), no solo en lo que concierne a las características de la escritura, sino también a su vinculación con el espacio de formación en el mundo digital o virtual. Sin embargo, argumentan disminuir el énfasis en las dos primeras (ubicuidad, temporalidad) y privilegiar aquellas referidas a componentes cognitivos y comunicativos dentro del proceso de escritura académica como la elaboración de tesis, como conclusión de la investigación en la cual diseñaron y analizaron las principales funciones de Thesis Writer, conceptualizado como un entorno de escritura bilingüe (alemán, inglés), basado en la nube, que conecta la escritura con la investigación, la colaboración y la supervisión a través de la enseñanza.

Con el propósito de desarrollar y fortalecer la alfabetización académica, algunas universidades han dado respuesta desde los Academic Skills Centres (Solimine y Marzal García-Quismondo, 2020); por ello, un CED puede hacer parte de una estrategia institucional más amplia, que aborde diferentes habilidades académicas. Por ejemplo, en Colombia, se encuentra el Centro de Recursos para el Éxito Estudiantil (CREE), de la Universidad del Norte, que además de tener un servicio de tutoría en la escritura, ofrece servicios en otras asignaturas y habilidades (Calle Álvarez, 2017). En la Universidad de Sídney, Australia, hay un centro de aprendizaje donde se acompañan habilidades como la escritura, el pensamiento crítico y la investigación. Otro ejemplo es el centro de enseñanza y aprendizaje de la Universidad de Japón, que acompaña habilidades en escritura, matemáticas y japonés (Okuda, 2020).

Lo anterior permite reafirmar que las decisiones en el diseño y la operación de un centro de escritura en la educación superior dependerán de los principios teóricos y necesidades prácticas de los centros educativos que lo fundamenten. Sin embargo, los atributos propios de los entornos digitales no pueden obnubilar el énfasis cognitivo, lingüístico y comunicativo propio de la escritura científica en el ámbito universitario, lo que amerita permanente estudio al respecto, que contribuya a fundamentar el diseño e implementación de estrategias experimentales para su fortalecimiento (Carpenter et al., 2017).

\section{Conclusiones}

Este estudio analizó la evidencia científica sobre la escritura académica universitaria, y mostró los resultados de un análisis sistemático (minería de datos) de 
investigaciones reportados por la literatura especializada, cuyo objetivo es ampliar su horizonte de comprensión y establecer las bases conceptuales orientadas al diseño de un CED.

Los aportes obtenidos contribuyen a fundamentar teóricamente la escritura académica para configurar e implementar un centro de escritura digital universitario, en el cual resulta necesaria la convergencia de postulados epistemológicos, relacionados con las formas de construcción del conocimiento; pedagógicos, asociados al propósito formativo que cumple la escritura en la ciencia y la cultura; y metodológicos, desde su integración con las tecnologías digitales.

Los resultados del corpus analizado muestran la consolidación de dos líneas de investigación para el campo de la escritura académica, el predominio de componentes pedagógico-didácticos para su promoción y el énfasis en el aprendizaje de la lengua materna respecto a lenguas extranjeras. Estos tres resultados evidencian no solo el interés de las instituciones universitarias por la enseñabilidad de la escritura, la cultura académica y la mediación de las tecnologías digitales, sino también por la necesidad de continuar investigando en este campo, para comprender y decidir, con base en resultados científicos, el diseño y la implementación de iniciativas al respecto.

Producto del análisis entre las dos líneas de investigación (centros de escritura vinculados el uso de las tecnologías digitales y comprensión de la alfabetización académica como generadora de pensamiento y conocimiento) es consecuente plantear que la generalidad de estudios analizados está dirigida a mejorar la escritura académica de los estudiantes universitarios, enfocadas en la adquisición de esta competencia desde el saber de la lengua, al margen del dominio del saber específico de las disciplinas. Una relación que debe ser bidireccional, particularmente para la producción y la divulgación del conocimiento científico.

Así, el diseño del CED se debería basar en la jerarquización de los componentes analizados, priorizando el componente pedagógico-didáctico, seguido del componente de formación disciplinar, el componente de comunidad virtual y finalmente el componente técnico, debido a las implicaciones del componente pedagógico-didáctico para la prestación de los servicios del CED. Los cuatro componentes deben estar en correspondencia con los postulados de la alfabetización académica, que brinda orientaciones para el acompañamiento personalizado a partir de tutorías individuales, el trabajo colaborativo mediante talleres de escritura que atienden a géneros discursivos o competencias específicas de quien escribe, y la estimulación de la autonomía cognitiva, por medio del uso de contenidos y estrategias disponibles en una biblioteca de recursos.

Estos hallazgos sugieren la importancia de profundizar en la comprensión y la dimensión formativa de la escritura académico-científica. Su significado contribuye no solo a fundamentar teóricamente un centro de escritura en el ámbito digital, sino también a su diseño e implementación, articulado a los principios de la formación universitaria (connotación filosófica de universalidad), su política lingüística y la gestión de propuestas formativas en modalidad virtual.

En el caso de la Universidad de Antioquia, donde se proyecta la experimentación empírica del CED en un estudio posterior (fase 2), a ella llegan estudiantes y profesores con diversas características culturales, sociales, cognitivas y de aprendizaje, y de alguna manera, las herramientas, estrategias y metodologías que se ofrecerán deberán estar en capacidad de responder a estas diversidades, aunque sea de modo parcial. El diseñar e implementar un CED que responda a las características de los pregrados y estudiantes de las seccionales de la Universidad de Antioquia visibilizará al departamento de Antioquia en una comunidad académica que se ha consolidado hace más de veinte años en relación con los centros de escritura. Estos, aún en escenarios digitales, representan una alternativa curricular transversal para materializar el aprendizaje colaborativo y contribuyen a conformar una 
comunidad virtual de aprendizaje, desde el CED, en la cual el intercambio con investigadores, escritores avanzados o pares académicos cualifica la propia formación como lector y escritor para la formación disciplinar científica o el ejercicio profesional. Esta condición promueve, en los estudiantes y profesores universitarios, una postura colaborativa en la producción y la divulgación de la ciencia, y además favorece el intercambio de información entre investigadores de diferentes latitudes.

\section{Agradecimientos}

Los autores extienden su agradecimiento a las entidades que hacen parte de la alianza para el desarrollo de los centros de escritura digital en la educación superior: Universidad de Antioquia (Colombia), Universidad Pedagógica de Heidelberg (Alemania) y Virtual Community Builder S. A. (Colombia).

\section{Referencias}

Al Murshidi, G. y Al Abd, K. (2014). UAE University students' awareness of using the writing center. Higher Education Studies, 4(3), 58-63. http://dx.doi. org/10.5539/hes.v4n3p58

Álvarez Pérez, P., López Aguilar, D. y Pérez Jorge, D. (2016). Programa de tutoría universitaria formativa y desarrollo del proyecto personal del alumnado. Revista de Pedagogía, 37(100), 67-89. https://www.redalyc. org/pdf/659/65949681005.pdf

Álvarez, G. y Difabio de Anglat, H. (2018). Retroalimentación docente y aprendizaje en talleres virtuales de escritura de tesis. Apertura, 10(1), 8-23. https:// dx.doi.org/10.32870/ap.v10n1.996

Amante Negrete, Y. M. y Gómez Zermeño, M. G. (2017). E-estrategias de lectura y escritura del inglés en ambientes virtuales de aprendizaje. Campus Virtuales, 6(1), 109-119. http://www.uajournals.com/ojs/index.php/campusvirtuales/article/view/194

Ballén Vargas, S. y Herrera González, A. F. (2017). Retos para los procesos de enseñanza y aprendizaje de la escritura académica en la Licenciatura en Lenguas Modernas (LLM) de la Pontificia Universidad Javeriana de Bogotá. Signo y Pensamiento, 36(71), 100-113. https://revistas.javeriana.edu.co/index. $\mathrm{php/signoypensamiento/article/view/21089}$
Barton, D. y McCulloch, S. (2018). Negotiating tensions around new forms of academic writing. Discourse, Context and Media, 24, 8-15. https://doi. org/10.1016/j.dcm.2018.01.006

Bleakney, J. (2019). Ongoing writing tutor education: Models and practices. En K. Gabrielle y T. Roggenbuck (Eds), How we teach writing tutors. Special issue of WLN Digital Edited Collection. https://wlnjournal. org/digitaleditedcollection 1/Bleakney.html

Broekhoff, M. (2014). A tale of two writing centers in Namibia: Lessons for us all. Journal of Academic Writing, 4(1), 66-78. https://doi.org/10.18552/ joaw.v4i1.92

Bucur, M. y Ban, A. (2019). The importance of the concept of communication among future engineers - a pre-test for a European study. Procedia Manufacturing, 32, 348-355. https://doi.org/10.1016/j. promfg.2019.02.224

CalleÁlvarez, G.Y.(2017).Perspectivadeloscentros deescritura en Colombia. Hallazgos, 14(28), 145-172. https:// dx.doi.org/10.15332/s1794-3841.2017.0028.07

Calle Álvarez, G. Y. (2019). Componente técnico para la estructuración de un centro de escritura digital. $\mathrm{Sa}$ ber, Ciencia YLibertad, 14(1), 311-323. https://doi. org/10.18041/2382-3240/saber.2019v14n 1.5232

Calle Arango, L. (2019). La pregunta en las tutorías de los centros de escritura. Íkala, Revista de Lenguaje y Cultura, 24(1), 137-152.https://doi.org/10.17533/ udea.ikala.v24n01a07

Calle Arango, L. (2020). Centros y programas de escritura en las IEs colombianas. magis, Revista Internacional de Investigación en Educación, 12(25), 77-92. https://doi.org/10.11144/Javeriana.m12-25.cpei

Calle Arango, L., Pico, A. L. y Murillo, J. H. (2017). Los centros de escritura: entre nivelación académica y construcción de conocimiento. Cadernos de Pesquisa, 47(165), 872895. https://dx.doi.org/10.1590/198053143882

Carlino, P. (2013). Escribir, leer y aprender a escribir en la universidad. Una introducción a la alfabetización académica. Fondo de Cultura Económica.

Carlino, P. (2017). Leer y escribir en las ciencias sociales en universidades argentinas. Enunciación, 22(1), 110124. https://doi.org/10.14483/22486798.12350

Carlino, P. (2018). Dos variantes de la alfabetización académica cuando se entrelazan la lectura y la escritura en las materias. Signo y Pensamiento, 36(71), 16-32. http://www.scielo.org.co/pdf/signo/ v36n71/0120-4823-signo-36-71-00018.pdf 
Carpenter, R., Whiddon, S. y Morin, C. (2017). "For writing centers, by writing centers": A new model for certification via regional organizations. WLN: $A$ Journal of Writing Center Scholarship, 42(1-2), 2-9. https://go.gale.com/ps/anonymous?p=AONE\&s $\mathrm{w}=\mathrm{w} \& \mathrm{issn}=\& \mathrm{v}=2.1 \& \mathrm{it}=\mathrm{r} \& \mathrm{id}=\mathrm{GALE} \% 7 \mathrm{CA} 5058$ $86197 \&$ sid $=$ googleScholar\&linkaccess $=$ fulltext

Casanovas Catalá, M. (2016). Las herramientas 2.0 en la escritura académica: buscadores y diccionarios. Folios, 1(43), 77-88. https://doi.org/10.17227/01234870 43 folios 77.88

Casanovas Catalá, M., Capdevila Tomás, Y. y Ciro, L. A. (2019).Literacidaddigitalyacadémica:contrastepreliminar entre dos universidades. Enunciación, 24(1), 87-102. http://doi.org/10.14483/22486798.13928

Chaverra, D. I. (Cood.). (2019). Aprender a escribir. Configuración de un centro de escritura digital en la enseñanza dela educación básica y media. Facultad de Educación, Universidad de Antioquia - Universidad Pedagógica de Heidelberg. http://bibliotecadigital.udea. edu.co/bitstream/10495/11473/5/ChaverraDoraInes_2019_AprenderEscribirConfiguracionCED.pdf

Chois-Lenis, P., Casas-Bustillo, A., López Higuera, A., Prado-Mosquera, D. y Cajas-Paz, E. (2017). Percepciones sobre la tutoría entre pares en escritura académica. Magis, 9(19), 165-184. https://www. redalyc.org/pdf/2810/281052678009.pdf

Clarence, S. y Dison, L. (2017). Writing centres in higher education. Working in and a cross disciplines. Africa Sun Media.

Clarence, S. y McKenna, S. (2017). Developing academic literacies through understanding the nature of disciplinary knowledge. London Review of Education, 15(1), 38-49. https://doi.org/10.18546/ LRE.15.1.04

Clerici, C. (2017). La presencia del otro como desafío de la escritura mediada por tecnología. ENSAYOS. Revista de la Facultad de Educación de Albacete, 32(1), 63-79. https://www.revista.uclm.es/index.php/ ensayos/article/view/1285

Crowley-Watson, M. (2019). Social media, aggregators, analytics, and the writing center. Communication Center Journal, 5(1), 162-166. http://libjournal. uncg.edu/ccj/article/view/1954

De Kerchov, D. (1999). Inteligencias en conexión. Hacia una sociedad de la web. Gedisa.

Elejalde Gómez, J. y Ferreira Cabrera, A. (2018). Modelo para un tratamiento de errores de transferencia en la escritura en ELE producida en comunidades virtuales.
Folios, (48), 137-152. https://doi.org/10.17227/ folios.48-8140

Fernández Jiménez, M., Mena Rodríguez, E. y Tójar Hurtado, J. (2017). Funciones de la tutoría en e-learning: estudio mixto de los roles del tutor online. Revista de Investigación Educativa, 35(2), 409-426. https:// doi.org/10.6018/rie.35.2.273271

Fernando, W. (2018). Show me your true colours: Scaffolding formative academic literacy assessment through an online learning platform. Assessing Writing, 36, 63-76. https://doi.org/10.1016/j.asw.2018.03.005

Figueroa, B. y Aillon, M. (2015). Escritura académica de un ensayo mediado por el aprendizaje colaborativo virtual. Estudios Pedagógicos, 41(1), 79-91. https://doi. org/10.4067/S0718-07052015000100005

Gainza, C. (2016). Prácticas académicas de producción y distribución del conocimiento en la era digital. (Y una reflexión sobre cómo transformarlas desde América Latina). Atenea (Concepción), (514), 263-278. https://dx.doi.org/10.4067/ S0718-04622016000200263

Gavari Starkie, E. I. y Tenca Sidotti, P. (2017). La evolución histórica de los centros de escritura académica. Revista de educación, (378), 9-29. https://dx.doi. org/10.4438/1988-592X-RE-2017-378-359

Graham, S. (2008). Strategy instruction and the teaching of writing. A meta-analysis. En: C. MacArthur, S. Graham y J. Fitzgerald (Eds.), Handbook of writing research (pp. 187-207). The Guilford Press.

Hermosillo, P. y Verdín, P. (2018). Perspectivas de la escritura académica en la universidad: causas, problemáticas y propuestas. Cuaderno de Pedagogía Universitaria, 16(31), 41-57. https://doi.org/10.29197/cpu.n31. v16.2019.04

Hernández Rojas, G. y Rodríguez Varela, E. I. (2018). Creencias y prácticas de escritura. Comparación entre distintas comunidades académicas. Revista Mexicana de Investigación Educativa, 23(79), 1093-1119. https://dialnet.unirioja.es/servlet/ articulo ?codigo $=7003756$

Iwasaki, C., Tada, Y., Furukawa, T., Sasaki, K., Yamada, Y., Nakazawa, T. e Ikezawa, T. (2019). Design of e-learning and online tutoring as learning support for academic writing. Asian Association of Open Universities Journal, 14(2), 85-96. https://doi. org/10.1108/AAOUJ-06-2019-0024

Jewitt, C. y Kress, G. (2008). Multimodal literacy. Peter Lang Publishing, Inc. 
Korenius, T., Laurikkala, J., Järvelin, K. y Juhola, M. (2004). Stemming and lemmatization in the clustering of finnish text documents. Thirteenth ACM international conference on Information and knowledge management (pp. 625-633). CIKM. https://doi. org/10.1145/1031171.1031285

Korotkina, I. (2017). Writing centers in Russia: Limitations and challenges. Working paper, Social Sciences Research Network. http://doi. org/10.2139/ssrn.2939495

Kozolup, M., Kokor, M. y Savchynskyi, R. (2020). Polish and Ukrainian university students' perspectives on academic writing: A comparative overview. Central European Journal of Communication, 13(3(27)), 352-370. http://doi. org/10.51480/1899-5101.13.3(27).3

Krüger, K. (2006). El concepto de la "sociedad del conocimiento". Biblio $3 W$, Revista Bibliográfica de Geografia y Ciencias Sociales, 11(683). http://www. ub.es/geocrit/b3w-683.htm

Kruse, O. y Rapp, C. (2019). Seamless writing: How the digitisation of writing transforms thinking, communication, and student learning. En: C. K. Looi, L. H. Wong, C. Glahn y S. Cai (Eds.), Seamless learning. Lecture notes in educational technology (pp. 191-208). Springer. https://doi. org/10.1007/978-981-13-3071-1_10

Latifi, S., Noroozi, O., Hatami, J. y Biemans, H. J. (2019). How does online peer feedback improve argumentative essay writing and learning? Innovations in Education and Teaching International, 58(2), 195 206. https://doi.org/10.1080/14703297.2019.16 87005

Limpo, T.y Alves, R. A. (2018). Effects of planning strategies on writing dynamics and final texts. Acta Psychologica, 188, 97-109. https://doi.org/10.1016/j. actpsy.2018.06.001

Lobato Fraile, C. y Guerra Bilbao, N. (2016). La tutoría en la educación superior en Iberoamérica: avances y desafíos. Educar, 52(2), 379-398. http://dx.doi. org/10.5565/rev/educar.726

López Gil, K. S. (2016). Lo que decimos sobre la escritura: caracterización de los recursos educativos digitales compartidos por centros y programas de escritura de Latinoamérica. Grafía, 13(1), 78-99. http://dx.doi. org/10.26564/16926250.657

López-Gil, K. S. y Molina Natera, V. (2018). Incidencia del trabajo colaborativo docente en la enseñanza y el aprendizaje de la escritura académica. Revista
Electrónica de Investigación Educativa, 20(1), 1-13. https://doi.org/10.24320/redie.2018.20.1.1477

Magnifico, A. M., Lammers, J. C. y Curwood, J. S. (2020). Developing methods to trace participation patterns across online writing. Learning, Culture and Social Interaction, 24. https://doi.org/10.1016/j. lcsi.2019.02.013

Magnifico, A. M., Woodard, R. y McCarthey, S. (2019). Teachers as co-authors of student writing: How teachers' initiating texts influence response and revision in an online space. Computers and Composition, 52, 107-131. https://doi.org/10.1016/j. compcom.2019.01.005

Maldonado, M. (2017). Escrituras académicas y literacidad: control de la escritura en la educación superior colombiana. Enunciación, 22(1), 68-82. https://doi. org/10.14483/22486798.10446

Márquez Guzmán, S. y Gómez Zermeño, M. (2018). Grupo virtual de escritura académica: una e-innovación para impulsar la publicación científica. Revista Mexicana de Investigación Educativa, RMIE, 23(76), 203-227. https://dialnet.unirioja.es/servlet/ articulo codigo $=6523380$

Márquez Jiménez, A. (2017). Educación y desarrollo en la sociedad del conocimiento. Perfiles Educativos, 39(158), 3-17. https://doi. org/10.22201/iisue.24486167e.2017.158.58635

Martínez Clares, P., Pérez Cusó, J. y Martínez Juárez, M. (2016). Las TICs y el entorno virtual para la tutoría universitaria. Educación XX1, 19(1), 287-310. https://doi.org/10.5944/educXX1.13942

Molina Natera, V. (2016). Capítulo XIII. Los centros de escritura en Latinoamérica: consideraciones para su diseño e implementación. En: G. Bañales Faz, M. Castelló Badía y N. A. Vega López (Coords.), Enseñar a leer y escribir en la educación superior. Propuestas educativas basadas en la investigación (pp. 339-362). http://web.metro.inter.edu/facultad/ esthumanisticos/crem_docs/Ensenar\%20a\%20 leer\%20y\%20escribir.pdf

Molina Natera, V. (2017). Resolución de conflictos en las tutorías de escritura según la teoría pragmadialéctica. Folios, (45), 17-28. https://doi.org/10.17227/01 234870.45 folios 17.28

Molina Natera, V. (2019). El discurso pedagógico en las tutorías de escritura. Develando elementos de una práctica educativa. Revista Mexicana de Investigación Educativa, 24(80), 125-148. http://www. scielo.org.mx/scielo.php?script=sci_arttext\&pid $=$ S1405-66662019000100125 
Molina-Natera, V. y López-Gil, K. S. (2020). Estado de la cuestión de los centros y programas de escritura de Latinoamérica. Revista Colombiana de Educación, 1(78), 1-25. https://doi.org/10.17227/ rce.num78-8066

Mora, R. A. y Golovátina-Mora, P. (2020). Video composition as multimodal writing: Rethinking the essay as post-literacy. KnE Social Sciences, 4(13), 4-12. https://doi.org/10.18502/kss.v4i13.7690

Moreno Mosquera, E. (2019). Lectura académica en la formación universitaria: tendencias en investigación. Lenguaje, 47(1), 91-119. https://doi.org/10.25100/ lenguaje.v47i1.7180

Munje, P. N., Nanima, R. D. y Clarence, S. (2018). The role of questioning in writing tutorials: A critical approach to student-centered learning in peer tutorials in higher education. Mentoring and Tutoring: Partnership in Learning, 26(3), 336-353. https://doi.org /10.1080/13611267.2018.1511953

Navarro, F., Ávila Reyes, N., Tapia-Ladino, M., Cristovão, V. L., Moritz, M. E., Narváez Cardona, E. y Bazerman, C. (2016). Panorama histórico y contrastivo de los estudios sobre lectura y escritura en educación superior publicados en América Latina. Revista Signos, 49(Supl. 1), 78-99. https://doi.org/10.4067/ S0718-09342016000400006

Navarro, F., Uribe Gajardo, F., Lovera Falcón, O. y Sologuren Insúa, E. (2019). Encuentros con la escritura en el ingreso a la educación superior. Representaciones sociales de los estudiantes en seis áreas de conocimiento. Ibérica: Revista de la Asociación Europea de Lenguas para Fines Especificos, (38), 75-98. https://dialnet. unirioja.es/servlet/articulo? codigo $=7203025$

Núñez Cortés, J. A. y Moreno Núñez, A. (2017). Percepción de los estudiantes universitarios iberoamericanos sobre la competencia comunicativa y la alfabetización académica. Zona Próxima, (26), 44-60. https:// dx.doi.org/10.14482/zp.26.10212

Okuda, T. (2020) The writing center and international students in a Japanese university: A language management perspective. Higher Education Research \& Development, 39(4), 778-791. https://doi.org/10.1 080/07294360.2019.1693515

Ortiz Casallas, E. M. (2015). La escritura académica en el contexto universitario (Pregrado). Zona Próxima, (22), 1-16. http://dx.doi.org/10.14482/zp.22.6076

Peña Portero, A. de la y Santamaría Martínez, R. (2020). Una intervención formativa del Centro de Escritura Nebrija en la Facultad de Ciencias Sociales.
Tendencias Pedagógicas, 36, 91-103. https://doi. org/10.15366/tp2020.36.07

Polizzi, G. (2020). Digital literacy and the national curriculum for England: Learning from how the experts engage with and evaluate online content. Computers \& Education, 152, 103859. https://doi. org/10.1016/j.compedu.2020.103859

Rodríguez Málaga, L., Rodríguez Pérez, C. y Fidalgo Redondo, R. (2019). Nuevos entornos de aprendizaje para la escritura: sistemas de tutoría inteligente. $\mathrm{Pa}$ peles del Psicólogo, 40(2), 133-140. https://dx.doi. org/10.23923/pap.psicol2019.2895

Romero González, A. N. y Álvarez Álvarez, M. (2019). Representaciones sociales de los estudiantes universitarios de grado sobre la escritura académica. Íkala, Revista de Lenguaje y Cultura, 24(1), 103-118. https:// dx.doi.org/10.17533/udea.ikala.v24n01a05

Salisbury, L. E. (2018). Just a tool: Instructors' attitudes and use of course management systems for online writing instruction. Computers and Composition, 48, 1-17. https://doi.org/10.1016/j.compcom.2018.03.004

Sánchez Ceballos, L. M. (2014). La escritura de calidad: base para la transformación de la instrucción en efectiva mediación didáctica. Revista Reflexiones y Saberes, 1(1), 32-37. https://revistavirtual.ucn.edu. co/index.php/RevistaRyS/article/view/512

Sharples, M. (1999). How we write. Writting as creative design. Routledge.

Sito, L., Méndez-Rendón, J. C. y Vásquez-Ramírez, L. Y. (2019). LEO en la práctica: la experiencia formativa en un centro de lecturas, escrituras y oralidades. Íkala: Revista de lenguaje y cultura, 24(2), 419438. https://dx.doi.org/10.17533/udea.ikala. v24n02a12

Solimine, G. y Marzal García-Quismondo, M. A. (2020). Proposal of visual literacy indicators for competencies courses. An academic literacy perspective for academic excellence. JLIS.it, 11(1), 17-35. https:// www.jlis.it/article/view/12577

Stella, J. y Corry, M. (2016). Intervention in online writing instruction: An action-theoretical perspective. Computers and Composition, 40, 164-174. http:// dx.doi.org/10.1016/j.compcom.2016.03.010

Strobl, C., Ailhaud, E., Benetos, K., Devitt, A., Krusse, O., Proske, A. y Rapp, C. (2019). Digital support for academic writing: A review of technologies and pedagogies. Computers and Education, 131, 33-48. https://doi.org/10.1016/j.compedu.2018.12.005 
Thompson, G. (2014). Moving online: Changing the focus of a writing centre. Studies in Self-Access Learning Journal, 5(2), 127-142.https://sisaljournal.org/archives/ june14/thompson/?shared=emailymsg=fail

Tiruchittampalam, S., Ross, A., Whitehouse, E. y Nicholson, T. (2018). Measuring the effectiveness of writing center consultations on 12 writers' essay writing skills. Languages, 3(1), 4. https://doi. org/10.3390/languages3010004
Vu, V., Warschauer, M. y Yim, S. (2019). Digital storytelling: A district initiative for academic literacy improvement. Journal of Adolescent \& Adult Literacy, 63(3), 257-267. https://doi.org/10.1002/jaal.962

Zárate Fabián, M. C. (2017). La escritura académica: dificultades y necesidades en educación superior. Educación Superior, 2(1), 46-54. http:// www.scivelo.org.bo/scielo.php?pid=S2518$82832017000100005 \&$ script $=$ sci_arttextv

Cómo citar este artículo: Chaverra, D. I., Calle-Álvarez, G. Y., Hurtado, R. D., y Bolívar, W. A. (2022). Revisión de investigaciones sobre escritura académica para la construcción de un centro de escritura digital en educación superior. Íkala, Revista de Lenguaje y Cultura, 27(1), 224-247. https://doi. org/10.17533/udea.ikala.v27n1a11 